\title{
¿Hacia una revalorización del trabajo remunerado de cuidados? Reflexiones sobre el Programa de Asistentes Personales del Sistema Nacional Integrado de Cuidados en Uruguay
}

\author{
Erika Van Rompaey \\ IICSAL-FLACSO/CONICET \\ evrgiuria@gmail.com
}

\section{Sol Scavino Solari}

Departamento de Sociología de la Facultad de Ciencias Sociales, Universidad de la República Oriental del Uruguay

Sol.scavino@cienciassociales.edu.uy

Artikulu honetan, Uruguaiko Zaintza Sistema Nazionalaren etxez etxeko laguntza-zerbitzuak eskaintzeko Laguntzaile Pertsonalen Programa aztergai hartzen da. Etxez etxeko zaintza-lanaren arloko lan-baldintzak aztertu nahi dira, eta baldintza horiek zaindua izateko eskubideari eta generodesberdintasunei nola eragiten dieten ezagutu. Aurkeztutako hipotesi nagusiaren arabera, etxez etxeko zaintza-lan ordainduaren ezaugarriak eta lanak dituen eskakizun tekniko altuak kontuan hartuta, horrelako prestazioak barneratutako arretaeredu publiko batek arta-lana egiten duten pertsonen berariazko prestakuntzaz gain, erakundeen sustengu teknikoa eskaintzea derrigorrezkoa da laneko esparru duinak eskaintzeko.

\section{GAKO-HITZAK:}

Arreta, lana, generoa, politika publikoak.
Este artículo analiza el Programa de Asistentes Personales, que brinda cuidados en domicilio para personas con dependencia severa del Sistema Nacional de Cuidados en Uruguay. Se busca examinar las condiciones en las que el trabajo de cuidados a domicilio es provisto y cómo ello impacta tanto en la garantía del derecho a ser cuidado, como en la reproducción de desigualdades de género. La hipótesis principal plantea que, dadas las características del trabajo remunerado de cuidados a domicilio y sus elevados requerimientos técnicos, un modelo público de atención que contemple este tipo de prestación requiere no solo de formación específica en cuidados de quienes realizan la atención, sino también de la provisión de soporte técnico institucional en el marco de condiciones laborales dignas.

\section{PALABRAS CLAVE:}

Cuidados, trabajo, género, políticas públicas. 


\section{Introducción}

La atención a las personas en situación de dependencia, entendida como "el estado en que se encuentran las personas que, por razones ligadas a la falta o pérdida de autonomía física, psíquica o intelectual, tienen necesidad de asistencia o ayudas importantes a fin de realizar los actos corrientes de la vida diaria", de acuerdo a la concepción adoptada en el Consejo de Europa -Resolución (98) 9, de 18 de septiembre de 1998-, ha estado ligada tradicional e históricamente a la vida íntima y privada de las personas, de las familias y, particularmente, de las mujeres. Las mujeres se han encargado de manera no remunerada de proveer los cuidados y cubrir las diversas necesidades derivadas de esta situación. Sin embargo, el fenómeno de la dependencia y, en particular, la cuestión de cómo resolver la provisión de atención y cuidados de esta población, han pasado a ser concebidos en las últimas décadas como problemas de interés público y colectivo.

Desde el mundo académico se han estudiado diversas dimensiones del cuidado en tanto actividad humana y, de esta, en tanto trabajo remunerado, examinándose algunas de sus características centrales y de las condiciones materiales e institucionales bajo las cuales es configurado y desarrollado. Por otro lado, la responsabilidad del cuidado recae fundamentalmente en las mujeres que lo llevan a cabo de forma no remunerada, así como también los trabajos remunerados de cuidados, típicamente femeninos, se caracterizan por sus malas condiciones laborales y desvalorización social. Dichos aspectos reflejan la producción y reproducción de las desigualdades de género, limitando las posibilidades de desarrollo de las mujeres.

En Latinoamérica, como en otras regiones del mundo, se encuentra una "crisis de cuidados". Es decir, tiene un conflicto social en relación a la provisión de cuidados, debido a un desfase entre la demanda y la oferta (Bazo, 1998; Durán 2006; Iglesias, 2006; Escudero, 2007). Esto se debe a una serie de factores sociales, demográficos, económicos y culturales, entre los que destacan los cambios en las familias, los procesos de envejecimiento poblacional y la lenta transformación de roles de género que expresa su impulso en la incorporación de las mujeres al mercado laboral, y su freno en la falta de corresponsabilidad en el trabajo doméstico y de cuidados por parte de los varones. Uruguay es uno de los países pioneros en la región en transitar un proceso de envejecimiento poblacional (Paredes et al., 2010). Tanto las tasas de natalidad como las de mortalidad son muy bajas, lo cual nos coloca como un país que ha experimentado lo que en demografía llaman "segunda transición demográfica”. Esta deja un saldo de personas que requieren cuidados en la vejez y, como se ha dicho antes, cada vez menos cuidadores, particularmente cuidadoras (Calvo et al., 2013), teniendo en cuenta que la población femenina económicamente activa aumentó del 40,4\% en 1986 al 55,6\% en 2013. La escasez de servicios públicos de cuidados, particularmente en las últimas dos poblaciones mencionadas en nuestro país es sorprendente. La atención para personas en situación de dependencia es profundamente insuficiente y es necesario avanzar en la creación y en la regulación de los servicios existentes, así como en la eventual clausura por incumplimiento de requisitos básicos (Mides, 2014).

En respuesta a este fenómeno, se ha creado recientemente el Sistema Nacional Integrado de Cuidados (SNIC), que pretende garantizar cuidados a la población dependiente y velar por los derechos de las personas a elegir ser o no cuidadoras. Asimismo, busca proporcionar las condiciones necesarias para llevar a cabo esa tarea, sin que sea un costo o un riesgo social asumido individualmente (Rodríguez Cabrero, 2011). El SNIC tiene dentro de sus objetivos modificar la actual división sexual del trabajo, en el marco de lo cual sus planes y programas deberían velar por la atención a las personas en situación de dependencia, a la población que las cuida, a la vez que fomentar cambios culturales en el cuidado para lograr una mayor corresponsabilidad entre los géneros.

Actualmente se están poniendo en marcha las primeras prestaciones orientadas a las personas con discapacidades en situación de dependencia y enfermos crónicos (particularmente personas mayores), a saber: la teleasistencia, el Programa de Asistentes Personales y los centros de larga estadía o de día.

Este artículo tiene el propósito de analizar las características del Programa de Asistentes Personales bajo la hipótesis de que, dadas las características del trabajo remunerado de cuidados en domicilio y sus elevados requerimientos técnicos (Van Rompaey, 2015), un modelo público de atención que contemple prestaciones para cuidados en domicilio donde el derecho a recibir cuidados sea garantizado, requiere no solo de la formación específica de quienes se ocupan en la atención directa de las personas dependientes, sino que además debe contemplar una organización y regulación institucional que brinde soporte técnico a quienes ejecutan la atención directa en el marco de condiciones laborales dignas.

Para ello, se reflexiona sobre el desafío de la política pública orientada a proveer atención a las personas en situación de dependencia en el Uruguay respeto a la protección del derecho a recibir cuidados, así como respecto a su capacidad para modificar los estereotipos y desigualdades de género, mediante el estudio de uno de sus principales programas de atención en domicilio, el denominado Programa de Asistentes Personales (AP).

\section{Las políticas de bienestar y de atención a la dependencia con perspectiva de género}

Entendido como una actividad indispensable para la reproducción humana, el cuidado tiene un rol central 
que es cada vez más reconocido y problematizado desde las ciencias sociales. Desde allí se lo ha señalado como una categoría analítica, a partir de la cual es posible explorar las formas que adquiere la división sexual del trabajo mediante el estudio de las conceptualizaciones de género subyacentes en los distintos estados de bienestar social y, en particular, de las políticas públicas orientadas a atender las necesidades de las personas en situación de dependencia, entre las que se encuentran los cuidados.

Así, y a pesar de las ambigüedades presentes en su definición, Daly y Lewis (2011) proponen que el cuidado puede significar una categoría de análisis de los Estados de Bienestar. Al respecto, plantean que a partir de la forma en la que los Estados conceptualizan y materializan (o no) los cuidados es posible conocer realmente la naturaleza de sus regímenes de bienestar (Daly y Lewis, 2011: 226). En este sentido, analizar el cuidado social ("social care"), permite la revisión de los derechos y los deberes de ciudadanía (Lewis, 1998, en Carrasco, Borderías y Torns, 2011) mediante el cuestionamiento y evaluación de los modelos sociales y las respuestas colectivas a la resolución del bienestar (Izquierdo, 2004; Vega, 2009; Tobío et al., 2010, en Carrasco, Borderías y Torns, 2011). Este enfoque supone que los Estados de Bienestar se enfrentan el desafío de lograr equidad en la participación de los distintos sectores que son fuente proveedora de bienestar (Estado, mercado, familia y comunidad) y entre varones y mujeres. Esto exige el diseño de una nueva organización social del cuidado que abogue por el estímulo a la corresponsabilidad (Adelantado et al., 1998; Daly y Lewis, 2000). En base a esta idea se han enunciado varias caracterizaciones a partir de cómo se organiza el cuidado entre los actores proveedores de bienestar. Por ejemplo, Torns (2008) y Martinez Buján (2010) recuerdan la distinción entre los modelos de bienestar y los modelos de cuidado. En los primeros, las políticas públicas de bienestar se generan en torno a los derechos de los trabajadores del mercado de empleo, a través de los cuales se produce el acceso a los derechos para el resto de los integrantes de las familias. Los segundos proponen universalizar sistemas de cuidado para atender a las personas dependientes y a los cuidadores, dado que consideran el cuidado como un derecho de ambas partes e independiente de la situación de las personas respecto al mercado laboral (Torns, 2008; Williams y Gavana, 2008, en Martínez Buján, 2010). Aguirre (2003) clasifica a los Estados como familistas (la responsabilidad del bienestar es de las familias y las mujeres) y desfamiliarizadores (hay una derivación de funciones de cuidado, tareas domésticas hacia el Estado y mercado). Este último modelo permite pensar la corresponsabilidad entre Estado, mercado, comunidad y hogares, en la realización de las tareas necesarias para proveer de bienes y servicios a la población pero particularmente para generar una nueva organización social del cuidado. Según Martínez y Voorend (2013), el primer tipo de Estados clasificados por Aguirre son los preponderantes en Latinoamérica. Lejos de estimular la corresponsabilidad entre sectores, los Estados en la región cuestionan poco el rol de las mujeres en el trabajo no remunerado de cuidados o directamente se basan en él para la aplicación de las políticas públicas y la generación de productos necesarios para el bienestar de la población.

Por otra parte, existen distintas perspectivas acerca de qué es la discapacidad y cómo se responde socialmente a las situaciones de dependencia derivadas de ella ${ }^{1}$. Grosso modo, siguiendo a Jiménez y Huete (2010) se podría decir que existen tres paradigmas en el concepto de discapacidad (con dependencia). El primer paradigma es el tradicional, del sometimiento o la marginación. En este, las personas con discapacidad (homologadas a personas con dependencia) son entendidas como excepciones, vinculadas a lo mítico y las respuestas sociales se orientan a ocultar o suprimir una desviación no deseada, lo cual deviene en el aislamiento y posición de marginación, inferioridad y rechazo. El segundo paradigma es el médico o de la rehabilitación. Este explica la discapacidad como parte de los elementos biológicos y se centra en las deficiencias y dificultades del individuo. En este caso las respuestas sociales se basan en rehabilitar y las personas con discapacidades son vistas como objetos pasivos sobre los cuales se actúa de manera técnica sin tener en cuenta los deseos o preferencias que estas podrían tener en tanto sujetos. El último es el paradigma social, de la autonomía personal o vida independiente. La explicación de la discapacidad se vincula con elementos relacionales y el problema se ubica en la sociedad, deslocalizando de los individuos. En este caso las respuestas sociales buscan equiparar oportunidades, eliminar la discriminación y la remoción de las barreras que limitan la participación. Se hace énfasis en la importancia de la participación plena de las personas con discapacidades en la educación, empleo y vida ciudadana, reforzando el hecho de que estas son sujetos de derechos.

${ }^{1}$ La investigación académica se ha centrado más en la problematización del concepto de discapacidad que en el de dependencia. Más bien la noción de dependencia ha sido exclusivamente empleada como concepto "paraguas" para aludir a un tipo de población que es objeto de políticas públicas, como en el caso español, donde se utiliza para definir la condición de las personas que se encuentran en situación de pérdida o ausencia de autonomía para ejecutar las actividades elementales de la vida cotidiana. Dicha definición se encuentra en sintonía con lo sostenido por el Consejo de Europa, que describe la dependencia como "aquel estado en el que se encuentran personas que, por razones ligadas a la falta o pérdida de la autonomía física, psíquica o intelectual, tienen necesidad de asistencia y/o ayudas importantes para realizar las actividades de la vida diaria". De acuerdo con Torres Pérez (2005), la dependencia puede afectar a cualquier sector de población y a cualquier edad, pero fundamentalmente a las personas de edad avanzada, así como a quienes tienen discapacidades físicas, psíquicas, patologías mentales o déficits sensoriales. Así, cuando se habla de personas en situación de dependencia, se hace alusión a aquellas que tienen algún tipo de discapacidad funcional, es decir, una discapacidad que merma o impide la capacidad de realizar por sí solas las actividades (básicas y o instrumentales) de la vida. Por ello, no todas las personas con discapacidad presentan una discapacidad funcional, es decir, se encuentran en situación de dependencia, dado que muchas de ellas no requieren de la ayuda de otra persona para llevar a cabo las actividades de la vida. 
Desde la política pública se han generado categorías para clasificar a los sujetos en situación de discapacidad, estableciendo que hay quienes pueden llevar a cabo las actividades básicas de la vida diaria y quienes no, así como también hay personas que no pueden realizar actividades instrumentales, pero si realizar las actividades básicas. A partir de estas categorías se plantean niveles de dependencia, lo cual establece que no todas las personas en situación de discapacidad son dependientes.

En este marco, las políticas de atención a la dependencia pueden reproducir el familismo y la feminización del cuidado existente en la región o bien contribuir a modificar estos aspectos que van en detrimento de la autonomía de las mujeres y la equidad entre los géneros, así como pueden reproducir el paradigma de marginación o medicalista, o bien apuntar a un paradigma social y de la interdependencia destacando los componentes relacionales del cuidado y el hecho de que tanto cuidadores como cuidados son sujetos activos como sujetos de derechos.

Si las políticas de cuidado se plantean como políticas de atención a la dependencia, centradas en las personas que requieren apoyos para el desarrollo de las actividades básicas e instrumentales de la vida cotidiana y dejan de lado a la población trabajadora de cuidados (remunerada o no) y las condiciones en las cuales dicho cuidado es provisto, probablemente se reproduzca la desvalorización del trabajo de cuidados. Esto tiende a invisibilizar los elevados niveles de cualificación requeridos para cuidar a las personas en situación de dependencia, más cuando ello se lleva a cabo en el domicilio de estas (Van Rompaey, 2015). Asimismo, si las políticas no incluyen condiciones suficientes y necesarias para garantizar la calidad del cuidado provisto, difícilmente lograrán garantizar y hacer efectivo el ejercicio del derecho a ser cuidados en el ámbito del hogar.

\section{Cuidados como trabajo, trabajo femenino socialmente desvalorizado}

El cuidado es un concepto en discusión, aunque podrían encontrarse algunos elementos en común para su definición (Batthyány, 2015). Primero, es una actividad que implica prestar ayuda o brindar apoyo para la realización de las actividades diarias de la vida de las personas en situación de dependencia que, debido a alguna enfermedad, a la propia edad o a raíz de un accidente, no pueden valerse por sí mismas. Dicha actividad tiene varias dimensiones, como la material (implica costos de oportunidades, de tiempo, económicos) pero también la vincular (suele existir un nexo emocional entre cuidadores y cuidados que es reconocido por su importancia en la tarea).

Folbre (1990) fue pionera en definir el cuidado como un servicio basado en la interacción personal sostenida entre quien cuida y quien es cuidado (frecuentemente a través de la relación cara a cara) y está motivado (como mínimo en parte) por la preocupación por el bienestar de la persona cuidada. A nivel de su contenido, el cuidado comprende a las actividades orientadas a dar satisfacción a las necesidades tanto físicas como emocionales de las personas en situación de dependencia (Daly y Lewis, 2000).

Por otro lado, el cuidado ha sido y continúa siendo una actividad caracterizada por ser realizada por mujeres. Es comúnmente llevado a cabo en el ámbito doméstico sin percibir una remuneración a cambio. La responsabilidad sobre el cuidado de personas en situación de dependencia ha sido concebida como una condición que define a las mujeres como sujetos sociales, a la par que ha sido entendida como una limitación para el desarrollo de estas en relación con otras áreas de sus vidas tales como la laboral, profesional, política, recreativa, entre otras (Graham, 1983). Waerness (1984) sostiene que la adscripción de las funciones de cuidado a las mujeres, además del incuestionable hecho de que solo las mujeres pueden tener hijos, se fundaba en una percepción de que la mujer es más intuitiva y emocional que el hombre, razón por la cual poseía una "inclinación natural" para el cuidado. Asimismo, Hochschild (1995) captura la dualidad amor-responsabilidad implícita en el cuidado al definirlo como un "vínculo emocional, usualmente recíproco entre quien brinda cuidado y la persona que lo recibe, en el cual la primera se siente responsable por el bienestar del otro y realiza trabajo mental, emocional y físico requerido para satisfacer dicha responsabilidad" (1995: 333).

Pero la tendencia a la mercantilización del cuidado ha generado un nuevo espacio en el mercado laboral que ha sido ocupado mayoritariamente por mujeres que trabajan en otros hogares o en instituciones, y perciben un salario por ello (Aguirre, 2013).

El cuidado, al ser considerado como trabajo, supone la existencia de un vínculo emocional trascendente en la tarea que lo distingue de otro tipo de trabajo remunerados y de otros trabajos no remunerados (Aguirre et al., 2014).

El concepto de cuidados como trabajo remunerado ("caring work") presentado por Celia Davies (1995) plantea que este comprende una amplia gama de trabajos caracterizados por relaciones interpersonales y que se estructura en torno a diversas configuraciones. Diferenció tres grandes tipos de cuidado: caregiving, carework y professional care. Dar cuidado ("caregiving") hace referencia a la actividad de cuidado en general que se orientada hacia la provisión de cuidado como actividad en términos generales con independencia de quién, cómo y en qué condiciones sea realizada. La actividad de cuidado concebida como trabajo remunerado ("carework") abarca a todo el conjunto de trabajos remunerados de cuidados no 
profesionales, ya sea ligado a personas en situación de dependencia o no. Por último, el denominado cuidado profesional ("professional care") se refiere al trabajo remunerado de cuidado llevado a cabo de manera profesional, caracterizado por la formación específica y sistemática de sus trabajadores y trabajadoras.

Con relación al estudio de las características o los rasgos distintivos del trabajo remunerado de cuidados, diversos autores (Waerness, 1984; Hochschild, 1998; Cancian y Oliker, 2000; England, 2005, entre otros), comparten la idea de que el cuidado es una actividad generalizada debido a la naturalización del trabajo de cuidados como un trabajo que compete a las mujeres, como tarea reproductiva y apuntalado en el evento biológico de que las mujeres quedan embarazadas (Cancian y Oliker, 2000). En este sentido, el sistema de género en las sociedades occidentales actuales impacta en la desvalorización del trabajo de cuidados, al igual que otros trabajos en los que la participación de las mujeres es mayoritaria. La desvalorización del trabajo femenino suele expresarse en la falta de reconocimiento de la importancia de la tarea (desvalorización simbólica) y en una mala o una nula remuneración por la realización de dicha tarea (desvalorización económica). Al respecto, Kaplan (1987) y Davies (1995) sostienen que la desvalorización social de la actividad de cuidado es mayor y más evidente cuando esta es llevada a cabo de forma remunerada sin estar sujeta a control o regulación burocrática, tal como fue constatado por Waerness (1992) en su investigación sobre las trabajadoras domésticas remuneradas.

En cuanto a las características globales del trabajo remunerado de cuidados, diversos investigadores consideran que la desvalorización social del cuidado se traspasa al ámbito de las profesiones, donde las ocupaciones en el sector de los servicios personales en general, y de cuidados en particular, se caracterizan por ser poco reconocidas y valoradas (Gomez Bueno, Daune-Richard, 1995; Abbot, 1988; Cameron y Moss, 2007; Torns et al., 2009; Recio, 2014).

En relación con el estudio del contenido del trabajo remunerado de cuidados, James (1992) distingue dos grandes dimensiones, la física y la emocional. La primera tiene que ver con la atención a las necesidades fisiológicas de las personas atendidas (higiene, vestimenta, alimentación y traslado, entre otras) y la emocional está ligada al soporte emocional requerido por parte de las personas cuidadas, así como a la necesidad de elaborar estrategias de modificación de las expresiones emocionales en las propias situaciones laborales, y de autocuidado fuera del ámbito laboral para hacer frente a la alta carga emocional que el trabajo conlleva (Van Rompaey, 2015). Sobre esta última dimensión, es preciso señalar que es la cara más invisible del trabajo, dado que no suele encontrarse protocolizada ni explicitada en las pautas de actuación laboral, además de que suele ser concebida como la parte más ardua desde la perspectiva de las trabajadoras (Van Rompaey, 2015). Hochschild, fue la primera en definir al trabajo emocional como la actividad que "requiere inducir o suprimir sentimientos en orden de mantener la expresión externa que produce cierto estado de ánimo en los otros, en este caso, el sentirse cuidado de una forma agradable y segura" (1983: 7). El trabajo emocional ("emotional labour") es distinto del trabajo de las emociones ("emotion work") ya que el primero se realiza exclusivamente a cambio de una remuneración y está guiado por los imperativos y las normas de la organización o empresa. Diversos autores han estudiado los empleos de atención directa a personas, constando que sus principales riesgos laborales tienen que ver con la sobrecarga emocional que producen en sus trabajadores, quienes con frecuencia padecen del síndrome de quemazón emocional (“burnout”) (Brotheridge y Grandey, 2002; Zapf, 2002; Ortega y López, 2004).

Davies (1995), Recio (2014) y Van Rompaey (2015) señalan que el trabajo de cuidados a domicilio presenta un rasgo único, que es el aislamiento y la consecuente soledad en el ámbito laboral respecto a otros trabajadores/as. Asimismo, constituye un trabajo altamente demandante (Davies, 1995), en tanto que implica lidiar con la incertidumbre asociada a la imprevisibilidad y gravedad de las situaciones que suelen emerger cotidianamente en la vida y el hogar de quienes son cuidados.

Según Van Rompaey (2015), los requerimientos técnicos (Littler, 1982) ligados al trabajo remunerado de cuidados en domicilio son elevados. Ello debido a los altos grados de responsabilidad y autonomía necesarios para dar satisfacción y resolver (parcial o completamente) las necesidades y problemas que emergen en el domicilio de las personas atendidas, así como de la variedad de competencias técnicas específicas que son efectivamente movilizadas durante el desempeño laboral. Dichas competencias pueden distinguirse en dos grandes tipos: por un lado, un conjunto que forma que incluye un acervo de saberes o conocimientos teórico-prácticos sobre salud y enfermedades, así como sobre técnicas de movilización e higiene, que son herramientas imprescindibles para atender a personas en situación de dependencia. La mayor parte de ellas solo puede ser adquirida mediante la formación específica y su consecuente puesta en práctica a través de la experiencia de trabajo concreta. Por otro lado, un grupo de competencias laborales denominadas "interpersonales", como la capacidad de comunicar, establecer empatía, la escucha atenta, la integridad ética, la capacidad para tomar decisiones, entre otras donde el rol de la formación en cuidados es insustituible como fuente de adquisición de la pericia requerida en el puesto de trabajo.

El trabajo de cuidados en domicilio conlleva riesgos laborales específicos que pueden ser categorizados en dos grandes grupos (Van Rompaey, 2015). Por un lado, los riesgos asociados a la alta carga física derivados de la atención de las necesidades 
fisiológicas de las personas usuarias del servicio (movilización y traslados, higiene, vestimenta, alimentación, etc.) como los dolores de columna y cervicales, rodillas, cansancio extremo, insomnio $y$, por otro, los riesgos vinculados a la alta carga emocional del trabajo efectivamente llevado a cabo. La sobrecarga emocional suele estar vinculada a la emergencia de problemas como la depresión y el síndrome de quemazón emocional, entre los más destacados. La dimensión emocional y la sobrecarga que para quienes realizan la tarea de cuidados supone, son aspectos que se encuentran escasa 0 no lo suficientemente abordados en el marco del soporte provisto por las organizaciones que brindan el servicio en ambos casos estudiados. Esto se deriva de la invisibilización de la dimensión emocional asociada al trabajo remunerado de cuidados, la cual no se encuentra protocolizada ni explicitada dentro de los márgenes de acción o actuación de quienes son responsables de la provisión del servicio de atención.

Dadas las características antes analizadas y siguiendo la línea de lo que plantea Torns (2008), para revalorizar el cuidado como un trabajo es preciso visibilizar y reconocer sus elevados requerimientos técnicos y generar condiciones laborales dignas que permitan profesionalizar servicios domésticos y de atención a la vida diaria. Ello permitiría revalorizar empleos altamente feminizados que se destacan por su precarización, informalidad y simbolización asociada al servilismo (Torns, 2008).

\section{La creación del Sistema Nacional Integrado de Cuidados como respuesta pública al problema de los cuidados}

En Uruguay, en respuesta a la crisis del cuidado se ha creado recientemente el Sistema Nacional Integrado de Cuidados (SNIC). Se habla de crisis de la demografía como un aumento de la población dependiente (principalmente personas mayores) en relación con las personas no dependientes, una sobredemanda de cuidados en relación con una escasa oferta de personas cuidadoras. Un constante aumento de la "brecha de cuidados" (Pickard, 2015).

En términos sociológicos, de manera poco exhaustiva, podría decirse que la crisis de cuidados responde a cambios en la concepción de las familias, cambios de género (entre ellos el aumento de la población económicamente activa femenina, y una carga de doble trabajo femenino que dificulta e impide el ejercicio de derechos ciudadanos, limita las oportunidades de desarrollo en distintos ámbitos generando condiciones de desigualdad entre varones y mujeres), cambios en los valores y prácticas en torno a lo colectivo y lo individual (procesos de individualización), así como al valor de la vida y su duración.

Respecto a esto último, Durán (2000) señalaba que la proporción de enfermos con relación a las personas sanas tiende a aumentar, lo cual se vincula con que los avances técnicos de la medicina han apuntado a alargar la vida pero no a hacerla más saludable.

El progresivo envejecimiento de la población uruguaya, evidenciado por el aumento de la proporción de personas de 65 años o más, que pasó del 2,5\% en 1908 al 13,4\% en 2014 (Paredes, Ciarnello y Burnet, 2010: 15), trajo aparejado que la sociedad tenga que hacer frente al aumento de la población dependiente. Si bien no todas las personas discapacitadas o mayores están en situación de dependencia, una parte de ellas necesita ayuda para realizar las actividades básicas de la vida diaria.

Frente al aumento de las personas dependientes, la respuesta a las necesidades de cuidado ha recaído $\mathrm{y}$ ha sido asumida por las mujeres de las familias otorgando cuidados no remunerados. No solo han asumido los cuidados dirigidos a las personas dependientes por la disminución de su autonomía para realizar actividades de la vida cotidiana por motivos físicos y mentales, sino también el cuidado que los niños y niñas requieren para su desarrollo.

Así lo han mostrado las Encuestas de Uso del Tiempo, cuya última medición en Uruguay (2013) muestra que en los hogares en donde se convive con niños menores de 12 años, 8 de cada 10 mujeres cuidan de manera no remunerada un promedio de 22 horas semanales, mientras que lo hacen 6 de cada 10 varones, $y 7$ horas de promedio menos que las mujeres (Batthyány, 2015: 99). En los hogares en los que hay presencia de personas con discapacidad dependientes, también cuidan 8 de cada 10 mujeres un promedio de 47 horas semanales y 5 de cada 10 varones, 49 horas semanales. En el caso de las personas mayores dependientes, los datos son débiles por la cantidad de casos, pero se estima que cuidan 6 de cada 10 mujeres, mientras que lo hacen 4 de cada 10 varones (Batthyány, 2015: 99). También, a nivel nacional la Encuesta de Cuidados No Remunerados en Salud ha mostrado que, de las personas que brindan cuidados no remunerados a personas con enfermedades crónicas o agudas, el $77 \%$ son mujeres y dedican 56 horas semanales promedio a la realización de dichas actividades (Batthyány, Genta y Perrotta, 2017: 205).

En este contexto, debido a la articulación de la generación de conocimiento por parte del mundo académico, la politización del problema promulgada por las organizaciones de la sociedad civil y los actores de gobierno, el cuidado ha tomado un lugar en la agenda pública uruguaya, en la cual desde 2010 se ha planteado la creación de un Sistema Nacional Integrado de Cuidados (SNIC), con el objetivo de redistribuir el trabajo de cuidados entre familia, Estado, comunidad y mercado, y entre varones y mujeres (Aguirre et al., 2014).

El SNIC se ha gestado durante un largo tiempo de discusiones políticas y sociales y en 2015 se publicó el Plan Nacional de Cuidados a ejecutarse 
desde 2016 hasta 2020. En él se establecen los objetivos del sistema, las poblaciones objetivo con las que trabajará y qué acciones se prevén para el mencionado período.

Dentro de los objetivos que el SNIC se plantea, aparecen como objetivo general:

"Garantizar el derecho de las personas en situación de dependencia a recibir cuidados en condiciones de calidad e igualdad, promoviendo el desarrollo de la autonomía, la atención y asistencia a las personas en situación de dependencia, así como el desarrollo infantil, en el marco de un modelo de corresponsabilidad entre familias, Estado, mercado y comunidad, así como entre varones y mujeres" (SNIC, 2015: 11).

Para dar cuenta de él, se plantean como objetivos específicos el aumento de la cobertura y mejorar la calidad de servicios para la primera infancia, mejorar la atención de personas mayores y con discapacidad en situación de dependencia, el fortalecimiento de capacidades institucionales y su mejora continua, la constitución de un marco legal para que se reconozca el derecho a cuidar y ser cuidado en condiciones de calidad, el desarrollo de oferta de formación para el trabajo de cuidados, la producción y gestión de la información oportuna y pertinentes sobre los sujetos de la política y las prestaciones y efectos del SNIC, así como la generalización del reconocimiento público del derecho a los cuidados mediante una estrategia comunicacional que contribuya a generar una transformación cultural para un mayor compromiso de corresponsabilidad social en los cuidados (SNIC, 2015: 11).

En el mismo plan, el sistema toma una definición de dependencia amplia en la que integra la situación de niños/as y de personas mayores y discapacitadas en situación de dependencia. Esta definición establece que son personas dependientes aquellas que "requieran apoyos específicos para el desarrollo de sus actividades y la satisfacción de las necesidades básicas e instrumentales de la vida diaria" (SNIC, 2015:8).

En este marco, el SNIC se propone generar servicios y prestaciones de cuidados para satisfacer las demandas de cuidado de la población no cubiertas por el Estado, con el fin de aliviar la carga de cuidados de las familias. Estos difieren según la población objetivo. Para hacer frente al déficit de opciones de cuidado infantil, se plantea el aumento de la cobertura de centros de cuidado, fundamentalmente de los que trabajan con niños de o a 3 años, donde hasta ahora la cobertura es escasa y mayoritariamente privada (Batthyány, Genta y Scavino, 2016).También se busca fomentar la utilización por parte de varones de las licencias parentales y mejoras en sus condiciones. Se plantea la creación de casas comunitarias de cuidados y la realización de transferencias económicas como las becas de inclusión socioeducativas, que permiten la articulación entre algunas actividades de la madre y los cuidados de los niños/as.

Por otra parte, los servicios y prestaciones que se dirigen a la población con discapacidad y personas mayores en situación de dependencia son de menor variedad, y se plantean en un escenario de mayor carencia de servicios preexistentes con relación a los centros de cuidado infantil. En Uruguay, el servicio de acompañantes, así como la posibilidad de contar con centros de larga estancia para el cuidado de personas dependientes, está fuertemente segmentado por el poder adquisitivo de los hogares, ya que la oferta es escasa, privada y cara. Según los datos del censo de 2011, se estima que el $2,5 \%$ de las personas mayores vive en centros de larga estancia. Desde el Instituto de las Personas Mayores del Ministerio de Desarrollo Social se lleva a cabo un censo de estos establecimientos para su identificación y regularización (se declara una gran presencia de centros de larga estancia de carácter informal en el ámbito privado). Los primeros resultados han mostrado la existencia de 1.030 centros en Uruguay, de los cuales el $75 \%$ son residenciales, el $7 \%$ hogares sin ánimo de lucro, el $5 \%$ servicios de inserción familiar y un $13 \%$ restante que no se clasifica en ninguna de las categorías anteriores (SIVE, 2015).

En el contexto de informalidad de los servicios y de su privatización, el derecho a recibir cuidados por parte de las personas mayores, y probablemente suceda lo mismo con las personas discapacitadas en situación de dependencia, está sujeto a la posibilidad de contratación de los hogares y, en los casos de contratación de servicios informales, puede llegar a implicar un riesgo de vida para estas personas $^{2}$.

Las prestaciones que el SNIC se plantea ofrecer específicamente a personas en situación de dependencia, entendiéndose como "el estado en que se encuentran las personas que, por razones ligadas a la falta o pérdida de autonomía física, psíquica o intelectual, tienen necesidad de asistencia o ayudas importantes a fin de realizar los actos corrientes de la vida diaria"2, consisten en: un programa de teleasistencia ${ }^{3}$, creación de centros de larga estancia y de día y reconfiguración del Programa de Asistentes Personales (Programa AP) para la atención de las personas discapacitadas que existía desde 2014 en la órbita del Banco de Previsión Social, asociado a las pensiones por discapacidad severa.

El Programa AP busca brindar apoyo a los familiares de personas en situación de dependencia. La asistencia personal se define como "el servicio que

\footnotetext{
${ }^{2}$ Por ejemplo, durante el mes de mayo de 2016, murieron 8 personas que vivían en un hogar de ancianos por un incendio. El hogar no contaba con la obligatoria habilitación de bomberos, que se había iniciado en 2014 http://www.elobservador.com.uy/el-hogar-el-quemurieron-7-ancianos-no-tenia-habilitacion-bomberos-n911365.

${ }^{3}$ Servicio dirigido a las personas con dependencia moderada que aún no se encuentra en funcionamiento.
} 
atiende el cuidado y la asistencia personal para las actividades de la vida diaria de las personas en situación de dependencia severa, es decir, que precisan ayuda para las necesidades básicas de la vida diaria (por ejemplo alimentarse, higienizarse, vestirse, movilizarse, trabajo, estudio y recreación, entre otras)" ${ }^{4}$.

Se trata de la presencia de un/a cuidador/a, que brinda ayuda profesional (se exige un curso de 90 horas proporcionado por el Sistema Nacional de Cuidados), pero que no puede suplir intervenciones profesionales. En los artículos publicados por el Ministerio de Desarrollo Social en su página oficial, se establece que uno de los objetivos principales "es priorizar las acciones de cuidado en domicilio con el objetivo de compartir las tareas de cuidado entre el Estado y las familias".

El Programa AP cuenta con dos tipos de componentes. Por un lado, una transferencia monetaria hacia las familias cuyos ingresos no excedan once BPC - Base de Prestaciones y Contribuciones- (36.740 pesos per cápita de ingresos mensuales) para el pago de un Asistente Personal. Transferencia que consiste en una liquidación y pago de 11.862 pesos uruguayos nominales 5 (unos 350 euros) por 80 horas mensuales de trabajo.

El segundo componente se orienta a la formación de asistentes personales a partir de un curso de 90 horas en total que los habilitará a realizar su trabajo. Asimismo, el programa crea un registro de asistentes personales habilitados que ofrece a las familias interesadas en ello. La elección del asistente personal puede ser por parte del beneficiario quien puede optar por contar con un asistente personal de la bolsa de trabajadores que se formarán desde el SNIC, o bien proponer a alguien con quien ya tenga una relación de cuidados para que pueda formarse como asistente personal.

Esta prestación es incipiente y plantea desafíos en materia de a quiénes se dirige, es decir, quiénes pueden acceder al subsidio económico, así como en qué condiciones se lleva a cabo el trabajo de cuidados remunerado en domicilio, en tanto que tiene efectos respecto a la modificación de la naturalización de los cuidados como una actividad típicamente femenina y socialmente desvalorizada. Estos aspectos serán abordados a continuación con el fin de poder dar cuenta de los elementos que favorecen o desfavorecen la protección del derecho a ser cuidado y la deconstrucción de los estereotipos de género asociados al feminizado y socialmente desvalorizado trabajo de cuidados.

4 http://www.sistemadecuidados.gub.uy/innovaportal/v/61052 /1/innova.front/asistentes-personales.

5 http://www.mides.gub.uy/innovaportal/v/63068/3/innova. front/es-hoy.

\section{5. ¿Cómo afecta la progresividad y la selección de la población objetivo del Programa AP a garantizar el derecho a recibir cuidados en domicilio?}

En su especificación se plantea que el Programa de Asistentes Personales está dirigido a apoyar en el cuidado de personas de entre o a 29 y 65 años o más que tengan discapacidad severa y que no vivan en hogares colectivos. Sin embargo, la inclusión de los beneficiarios es progresiva y en 2016 y 2017 podrán recibir asistentes personales del SNIC los menores de 29 años y mayores de 85 años, dejando fuera al resto de la población en situación de dependencia $y$, particularmente, a las personas mayores de entre 65 y 85 años, entre quienes las situaciones de dependencia para las actividades de la vida diaria son altas.

Según los datos de la Encuesta Longitudinal de Protección Social de $\mathbf{2 0 1 5}$ del Banco de Previsión Social, de las personas con discapacidad, el $45 \%$ manifiesta necesidad de un asistente personal por no poder realizar las actividades de la vida diaria. Sin embargo esto es distinto entre varones y mujeres según el tramo de edad. De los varones discapacitados que declaran necesitar atención personal, el 25,6\% tiene entre o y 25 años, mientras que el $38,9 \%$ son varones de 65 años o más. En el caso de las mujeres la proporción de discapacitadas que necesitan un asistente personal es mayoritaria en el caso de las mujeres mayores, de 65 años o más, ya que representan un $62,5 \%$ del total de las mujeres que precisan asistentes personales. Las que tienen entre o y 25 años representan el 12,4\% del total (BPS, 2015). A su vez, en un primer acercamiento a la medición de la dependencia realizado por dicha encuesta indica que un $14 \%$ de las personas de 65 años o más (cerca de 65.380 personas) manifiestan tener dificultad para realizar al menos una actividad de la vida diaria, de las cuales un $9 \%$ declara tenerlas siempre (BPS, 2015). Y si bien en el tramo etario de 85 años o más es donde hay mayor prevalencia de la dependencia (31,6\%, seguido del tramo de 74 a $85(16,7 \%)$ y siendo menor el porcentaje de personas con dependencia de 65 años a 74 $(8,3 \%)$, la cantidad de personas de 85 años o más es significativamente menor que la cantidad de personas entre 65 y 75 .

Es por esto que la progresividad de la prestación hace que, a dos años de la existencia del SNIC, el acceso de la población en situación de dependencia a la prestación se encuentre extremadamente limitada, dados los criterios de focalización en base a edad (no acceden quienes tienen entre 30 y 64 años) y grado de dependencia (solo para quienes tienen dependencia categorizada como severa). Ello pone en tela de juicio que el derecho a ser cuidado de las personas en situación de dependencia sea efectivamente garantizado por medio del Programa de Asistentes Personales. 
A lo anterior se añade un segundo motivo que limita el acceso a la prestación y merma la efectiva protección del derecho a ser cuidado. Como fue anteriormente señalado, el Programa AP considera el poder adquisitivo de los hogares para otorgar el subsidio económico para el pago de un asistente personal. El copago es progresivo con relación a los ingresos de los hogares: aquellos de mayores niveles de ingresos, pagan el $100 \%$ del costo. En este sentido, el tercer criterio de acceso a la prestación, dado por el nivel de ingresos de los hogares, comúnmente denominado sistema de copago de la prestación, desvirtúa la universalidad de la política y por tanto, pone en duda la consagración de la efectiva protección del derecho a ser cuidado. No percibe la prestación toda persona que por su condición de dependencia requiera de ayuda en domicilio y tenga derecho a ser cuidado, sino sólo aquellas personas cuyo ingreso por hogar no supere determinado nivel preestablecido.

Como resultado de la actual focalización del programa, en 2016 se estimó la atención a 3.500 personas con dependencia severa ${ }^{6}$, en el marco del establecimiento de un régimen de copago.

\section{6. ¿Cómo afectan las características del Programa AP a la desvalorización del trabajo de cuidados en domicilio?}

Para poder analizar las condiciones del trabajo de las asistentes personales del Programa AP y cómo impactan en la desvalorización del trabajo de cuidados en domicilio es preciso tener en cuenta cuáles son las características y requerimientos técnicos del trabajo de cuidados en domicilio. En este sentido, un aspecto que distingue al trabajo remunerado de cuidados en domicilio de otro tipo de trabajos remunerados de cuidados es la gran responsabilidad de quien cuida respecto al bienestar de la persona cuidada. Dicha responsabilidad es aún mayor, así como los márgenes de autonomía son elevados, cuando la atención y los cuidados son provistos en los domicilios de las personas cuidadas, dado que la atención implica la resolución de muchos de los problemas que emergen en el domicilio, donde sucede la vida íntima de las personas cuidadas. Todo ello debe ser asumido por quienes cuidan de manera remunerada en un contexto de aislamiento físico y soledad respecto a otras personas que realizan la atención directa (Van Rompaey, 2015).

De manera más específica, el trabajo de cuidados en domicilio consta de dos dimensiones centrales que estructuran las prácticas y rutinas de trabajo diarias: la física y emocional. La primera implica brindar soporte físico para la realización de las actividades básicas de la vida diaria (vestirse, asearse, alimentarse, trasladarse, etc.) y la cara más visible

6 http://www.mides.gub.uy/innovaportal/v/63068/3/innova. front/es-hoy. (y usualmente explicitada y regulada del trabajo). La segunda supone proveer de soporte emocional a las personas atendidas (escucharlas, animarlas, consolarlas, etc.), así como elaborar estrategias de autocuidado dentro y fuera del ámbito laboral, siendo la cara más invisibilizada del trabajo. A su vez, la dimensión emocional del trabajo también requiere de la gestión o regulación de las propias emociones de las trabajadoras, tanto en el puesto de trabajo como fuera de él. Como bien indicaba Hochschild, la regulación de las expresiones externas de las emociones en las situaciones de trabajo, pero sobre todo la regulación interna de las emociones en tanto aspecto fundamental e imprescindible para hacer frente a las consecuencias negativas asociadas a la alta carga emocional que supone lidiar con personas que padecen de múltiples problemáticas ligadas a su condición de dependencia.

De ambas dimensiones del trabajo señaladas devienen riesgos laborales específicos. Las tareas físicas que las trabajadoras tienen que realizar para brindar soporte físico a las personas dependientes suelen traer aparejadas problemas de salud entre los que suelen encontrarse los dolores de espalda y cervicales, de rodillas y cansancio extremo, entre otros (Van Rompaey, 2015). Vinculados a la dimensión emocional del trabajo aparecen riesgos laborales específicos ${ }^{7}$ (Hochschild, 1983; Van Rompaey, 2014) devenidos de la sobrecarga emocional que implica actuar como soporte emocional de las personas cuidadas, así como de la acumulación de sensaciones de desagrado, asco, miedo o impotencia derivadas de las tareas vinculadas al cuerpo, enfermedades o limitaciones de la personas atendida. Ello provoca la consecuente necesidad de llevar a cabo esfuerzos para regular las propias emociones, requeridos para poder afrontar el cuidado y la resolución de problemáticas que emergen en el domicilio y que suelen ser imprevisibles, así como para dejar de lado dichas emociones una vez terminado el trabajo.

Por último, este tipo de trabajo requiere de una amplia formación específica en cuidados que permita dotar a las trabajadoras de herramientas tanto teóricas (sobre salud en general y enfermedades específicas en particular) como prácticas (técnicas

${ }^{7}$ Hochschild (1983) sostiene que, a diferencia del trabajo arquetípico en el modelo industrial moderno, los trabajos remunerados que implican la atención directa a usuarios o clientes, ya sea bajo la modalidad cara a cara o voz a voz, dada su naturaleza relacional, exponen en mayor medida a sus trabajadores/as a las consecuencias negativas o costos asociados al trabajo emocional. Plantea que, cuando la expresión emocional es parte esencial del servicio provisto durante largo tiempo por el o la trabajador/a, suele generarse una separación entre la expresión de los sentimientos fingidos y los sentimientos realmente vividos internamente. Cuando esto sucede, el principio de disonancia emocional, análogo al de la disonancia cognitiva, comienza a operar y mantener la disonancia emocional entre lo que se siente y lo que se finge por un largo periodo provoca estrés. Las personas tienden a reducir dicho estrés al procurar acercar ambas cuestiones, cambiando lo que sienten o lo que fingen sentir. Cuando una determinada expresión es requerida en el puesto de trabajo, es usualmente el sentimiento el que tiene que cambiar, y cuando las condiciones nos extrañan respecto a nuestras caras, nos extrañan también respecto a lo que sentimos. 
de higiene, movilización, etc.) para poder realizar el trabajo de manera adecuada, mitigando los riesgos laborales propios del puesto de trabajo.

A continuación se analizan las principales condiciones en las que el trabajo de cuidado en domicilio es provisto en el marco del Programa AP y cómo inciden en favorecer o no la desvalorización del trabajo de cuidados.

\subsection{Insuficiente formación}

Según se observa en los planteamientos del Programa de Asistentes Personales propuesto por el SNIC, está diseñado desde una lógica de atención a la dependencia, individualizada, en la cual el sistema provee de un subsidio para el pago de trabajadoras/ es por parte de los hogares, "calificados" para el cuidado con cursos de 90 horas semanales, siendo la exigencia del nivel educativo para quienes cuidan tener primaria completa. Por un lado, dicha formación específica y requerida para acceder al trabajo remunerado de cuidados a domicilio es insuficiente para dotar de herramientas adecuadas a quienes se ocupan en este tipo de empleo. Ello teniendo en cuenta que en el caso barcelonés, las trabajadoras en domicilio requieren para acceder al empleo de una formación básica o mínima en cuidados de 450 horas, es decir cuatro veces más que las que se plantea el Programa AP, la cual además consideran insuficiente para poder llevar a cabo un desempeño laboral adecuado en el domicilio (Van Rompaey, 2015).

\subsection{Falta de soporte técnico y mecanismos de prevención de riesgos laborales}

Por otro lado, tal como está diseñado el Programa AP, no se prevé ningún tipo de soporte técnico a las trabajadoras que realizan el trabajo de cuidados en domicilio. En este sentido, las asistentes personales no cuentan con una coordinación de referencia que pueda apoyar y asistir técnicamente a quienes realizan la atención directa cuando se presentan casos o situaciones complejas con las personas atendidas, así como tampoco cuentan con el soporte de un seguimiento técnico de los casos atendidos a lo largo del tiempo. Asimismo, tampoco se establecen mecanismos de prevención de riesgos laborales para un trabajo que requiere de gran responsabilidad y capacidad de toma de decisiones, donde las trabajadoras se encuentran aisladas y deben asumir de manera solitaria la gran tarea de hacerse cargo del bienestar de las personas atendidas (reuniones grupales de equipo, formación en estrategias de autocuidado, etc.).

Dado lo anterior, se considera que el programa no contempla ninguna acción que tenga en cuenta que el trabajo de cuidados es un trabajo con altos riesgos a nivel emocional y físico por parte de los y las trabajadores, lo cual también implica una reproducción de las malas condiciones laborales que ya tienen los cuidadores remunerados formales 0 informales en Uruguay.

\subsection{Bajo salario}

El salario que se brinda a los cuidadores/as -asistentes personales- es muy bajo, si se considera lo establecido como salario mínimo nacional (11.50o pesos en 2016). De esta manera, el trabajo de cuidados es mercantilizado por el propio Estado como un trabajo de los peor pagados. Esto refuerza un fenómeno que ya sucede con el trabajo doméstico y de los cuidadores a domicilio remunerados en nuestro país, representándolo como un trabajo de escaso valor. En vez de contribuir a que los salarios aumenten, a que los cuidados se valoren como empleo y se reconozca a través de la paga el fundamental aporte que supone para el sostenimiento de la vida, la capacidad de que las sociedades se reproduzcan y subsistan y su aporte al bienestar social.

El punto de partida en el trabajo de cuidados es que carece de valor social, asociado al servilismo y naturalizado como una tarea de las mujeres en el marco de las familias. Tal como se ha indicado más arriba, las encuestas de uso del tiempo indican la desvalorización de este trabajo en Uruguay, ya que se realiza mayoritariamente de manera gratuita. Es decir, que mediante a él no se accede a un salario. Asimismo, cuando las personas trabajan en el mercado de empleo ofreciendo cuidados, adquieren sueldos muy bajos y tienen trabajos mayoritariamente informales, que no les permiten acceder a derechos laborales y sociales básicos. Pero el estímulo de la contratación de personal doméstico a domicilio que se encuentra en el Programa de Asistentes Personales podría significar una fuente de revalorización y reconocimiento de este trabajo si fuese muy bien pagado. No obstante, se considera que la propuesta de asistentes personales del SNIC no contribuye a crear mejores condiciones de empleo en el sector de los cuidados, sino que se limita a imitar las condiciones de trabajo del sector cuidados en Uruguay.

Como muestra la Tabla 1, estas condiciones son informales y precarias. En primer lugar, el sector de los cuidados está altamente feminizado (del total de los trabajadores, el $94 \%$ son mujeres). El $96 \%$ de trabajadores que realizan tareas similares a las de asistencia personal son mujeres con un promedio de edad más elevado con relación a la población ocupada total (50 años, frente a 41)

Las condiciones de informalidad son frecuentes en el sector de los cuidados y se reflejan a través de indicadores como el aporte a la caja de jubilaciones (aproximadamente 6 de cada 10 trabajadores de cuidados similares a los asistentes personales declara no aportar a la caja, mientras que para el total de la población ocupada esta cifra se acerca 
casi a 3 de cada 10). También en el hecho de que el $45 \%$ de los trabajadores de cuidados similares a los asistentes personales no cuenta con vacacionales pagadas por sus empleadores (el $86 \%$ declaran ser asalariados privados). Paradójicamente, la mitad de las personas que trabajan cuidando de personas dependientes no cuentan con baja por enfermedad o lesiones, mientras que lo hace el $89 \%$ de la población ocupada total.

Finalmente, dentro de los cuidadores que trabajan actualmente realizando tareas similares a las que harán los asistentes personales, se evidencia una mayor presencia de población afroamericana o negra en relación con la población ocupada total.

\subsection{Reproducción de los estereotipos de género en el cuidado naturalmente femenino}

El Programa de Asistentes Personales, dadas sus características, no plantea ningún estímulo específico para que la población cuidadora sea compuesta por varones y mujeres de similar manera, si tomamos como referencia la oferta de personas en el sector de los cuidados y las preferencias de la población uruguaya en el cuidado en niños y adultos mayores familiares y femeninas (Batthyány Genta y Perrota, 2013). Es importante recordar aquí que las familias pueden proponer a los asistentes personales, lo cual supone que la familia es el mejor actor para hacerlo, idea cuestionable debido a que no necesariamente las personas integrantes de las familias cuentan con la mejor información para elegir a las personas cuidadoras, siendo probable que se orienten por mujeres. Esto nos lleva al segundo punto, que trata sobre el estímulo al cambio en la actual división sexual del trabajo. Esta se expresa entre el trabajo remunerado y el no remunerado, pero también dentro del trabajo remunerado, donde se visualiza una fuerte feminización de los trabajos peor pagados, más precarios y de baja calidad pero fundamentales para el bienestar social como la educación, la salud o los cuidados (Inmujeres, 2013: 30-32). Así, la escasa presencia de varones en trabajos remunerados de cuidados contribuye a reforzar la feminización de la tarea y la reproducción del estereotipo de mujer como madre y naturalmente preparada para ser cuidadora. En este punto, es importante mencionar que a partir de la reproducción como fenómeno biológico (en el caso de las mujeres, la posibilidad de quedar embarazadas y amamantar), se han apuntalado representaciones sobre las mujeres como principales cuidadoras por "naturaleza". Esto, que parecería no vincularse con el cuidado de las personas en situación de dependencia más allá de los/as niños/as, se relaciona con el hecho de que en cierto punto de la historia occidental se necesitó crear una figura de persona cuidadora, para la cual la construcción de un tipo de maternidad tendió un puente casi indisociable entre mujeres y cuidados (Chorodow, 1978).

La construcción de madre buena por naturaleza, abnegada, paciente, disponible y cuidadora, en contraposición a la construcción de padre como ganador del pan, exitoso y sin sentimientos, se desarrollaron vinculadas a la idea de una familia

Tabla 1. Estadísticas descriptivas sobre la población empleada en el sector de los cuidados y características del empleo (2016)

\begin{tabular}{|c|c|c|c|c|}
\hline & & \multirow{3}{*}{$\begin{array}{c}\begin{array}{c}\text { Cuidadores } \\
\text { en general }\end{array} \\
6,0 \\
\end{array}$} & \multirow{3}{*}{$\begin{array}{c}\begin{array}{c}\text { Cuidadores } \\
\text { similares a } \\
\text { asistentes } \\
\text { personales }\end{array} \\
4,5 \\
\end{array}$} & \multirow{3}{*}{$\begin{array}{c}\begin{array}{c}\text { Ocupados } \\
\text { (Total) }\end{array} \\
55,2 \\
\end{array}$} \\
\hline & & & & \\
\hline \multirow{2}{*}{ Sexo } & Varón & & & \\
\hline & Mujer & 94,0 & 95,5 & 44,8 \\
\hline \multirow{2}{*}{ Principal ascendencia étnico-racial } & Afroamericana o negra & 4,9 & 5,5 & 4,3 \\
\hline & Indígena & 1,9 & 2,1 & 1,7 \\
\hline \multicolumn{2}{|l|}{ Edad (media) } & 42 & 50 & 41 \\
\hline \multirow{3}{*}{ Categoría de la ocupación } & Asalariado/a privado/a & 82,8 & 86,3 & 56,8 \\
\hline & Asalariado/a público/a & 7,1 & 0,4 & 15,0 \\
\hline & $\begin{array}{l}\text { Cuenta propia con o sin } \\
\text { local o inversión }\end{array}$ & 9,8 & 13,2 & 22,9 \\
\hline \multicolumn{2}{|c|}{ Reconocimiento de horas extras trabajadas (sí) } & 41,8 & 33,5 & 42,7 \\
\hline \multicolumn{2}{|c|}{ Vacaciones anuales pagadas o pago por permiso no disfrutado (sí) } & 58,0 & 45,1 & 62,7 \\
\hline \multicolumn{2}{|c|}{ Baja en caso de enfermedad o lesión (sî) } & 55,0 & 41,4 & 61,1 \\
\hline \multicolumn{2}{|l|}{ Cobra aguinaldo (sî) } & 63,2 & 50,9 & 65,1 \\
\hline \multicolumn{2}{|l|}{ Aporta a alguna caja de jubilaciones (sí) } & 55,2 & 40,8 & 74,7 \\
\hline \multicolumn{2}{|l|}{ Aporta por la totalidad del salario (sî) } & 51,0 & 36,6 & 61,0 \\
\hline \multicolumn{2}{|c|}{ Promedio de ingresos de la ocupación principal } & 12.896 & 11.147 & 25.871 \\
\hline \multicolumn{2}{|c|}{ Cantidad de horas promedio dedicadas al trabajo principal } & 33 & 36 & 38 \\
\hline
\end{tabular}

Fuente: Elaboración propia en base a los microdatos de la Encuesta Continua de Hogares, Instituto Nacional de Estadística, Uruguay, 2016.

Nota: Incluye los códigos 5311, 5312, 5321, 5322 y 5329 (cuidadores de niños, ayudantes de maestras, trabajador de cuidados personales en instituciones, trabajador de cuidados personales a domicilio, trabajadores de cuidados en servicios de salud. Se sigue la clasificación presentada por Aguirre (2014) [8]Se trata de los trabajadores de cuidados personales a domicilio (código 5322 del codificador de ocupaciones CIIU 08) Incluye: Cuidador de enfermos (domicilio), Acompañante de personas convalecientes (domicilio), ayudante de enfermería (domicilio), Camillero de ambulancia (domicilio). 
heteronormativa tipo (padre, madre e hijos de ambos) y contribuyeron a sostener no solo la expansión del capitalismo, sino también el lugar de poder de los varones en el ámbito público y político. Bock y Duden (1985) sostienen, de hecho, que es el "amor" de madre el que ha operado como ideología para el sostenimiento y desarrollo de este modelo socioeconómico.

En sus palabras, “el trabajo doméstico (de cuidados y doméstico) se escondería detrás de la noción de amor, mientras que "trabajo" vino a significar, además de algo masculino, el ganarse un salario fuera de casa. [...] La génesis del trabajo doméstico, como elemento esencial de la sociedad capitalista, solo quedó completa con su generalización y su extensión al conjunto de las mujeres" (Bock y Duden, 1985: 7). Este modelo patriarcal heteronormado generó pues un modelo específico de maternidad que se replica y expande a otras situaciones de cuidado.

La asistencia personal se plantea en el marco de los hogares, donde las relaciones de poder (ya sea por parte de los familiares del dependiente, de los dependientes o de los cuidadores) están presentes y son más difíciles de regular y controlar por agentes externos. La familia y el hogar aparecen como el entorno ideal para el cuidado sin ser problematizado, ni regulado por el programa. En este sentido, podrían existir protocolos de actuación de las trabajadoras a domicilio que pauten los límites respecto a las tareas a ser desarrolladas y las condiciones en las que estas pueden o no ser realizadas. Sin duda, el control de las relaciones laborales en el espacio doméstico, en el que puede darse una situación de abuso difícil de controlar por parte de los empleadores, y el estímulo de participación en el trabajo de cuidados por parte de varones, parece fundamental a la hora de proponer acciones de cuidados que busquen la incorporación de una perspectiva de género y la deconstrucción del estereotipo de mujer en tanto naturalmente dotada para los cuidados. Así, a pesar de que el Estado quiere responsabilizarse por alguna parte del cuidado (lo que indica luces en la intención y concienciación del problema por parte del Gobierno y el Estado), lo hace de manera tal que reproduce desigualdades de género y la falta de reconocimiento del valor social del trabajo de cuidados.
Tabla 2. Resumen de las dimensiones y características del Programa de Asistentes Personales
\[ \text { Características del trabajo de asistentes personales } \]

\begin{tabular}{|c|c|c|}
\hline & $\begin{array}{l}\text { Características del trabajo de asistentes personales } \\
\text { propuestas por el Sistema Nacional Integrado de } \\
\text { Cuidados (SNIC) }\end{array}$ & $\begin{array}{l}\text { Dispositivo previsto en el Programa de Asistentes } \\
\text { Personales }\end{array}$ \\
\hline \multirow[b]{2}{*}{$\begin{array}{l}\text { Grado de autonomía por parte } \\
\text { de las/os trabajadoras/es }\end{array}$} & \multirow{2}{*}{$\begin{array}{l}\text { El trabajo de asistente personal se plantea como } \\
\text { un trabajo autónomo por parte de los asistentes } \\
\text { personales en los hogares, el SNIC aparece como } \\
\text { "supervisor" del trabajo. Su función es la de } \\
\text { organismo al que acudir en caso de demandas o } \\
\text { conflictos. }\end{array}$} & $\begin{array}{l}\text { No está claro aún cómo será la supervisión de los } \\
\text { asistentes personales. }\end{array}$ \\
\hline & & $\begin{array}{l}\text { Para garantizar la autonomía vía el conocimiento } \\
\text { que manejan los trabajadores, el SNIC brinda un } \\
\text { curso de } 90 \text { horas en total para la formación de los } \\
\text { asistentes personales. }\end{array}$ \\
\hline Responsabilidad & $\begin{array}{l}\text { La responsabilidad física y psíquica del trabajo de } \\
\text { cuidados es asumida por el o la trabajadora de manera } \\
\text { individual y aislada. }\end{array}$ & $\begin{array}{l}\text { El SNIC no prevé mecanismos para colectivizar } \\
\text { la responsabilidad del trabajo de cuidados o } \\
\text { garantizar condiciones de trabajo menos riesgosas } \\
\text { en domicilio }\end{array}$ \\
\hline Riesgos laborales & $\begin{array}{l}\text { No se mencionan en el diseño del programa de } \\
\text { asistentes personales. }\end{array}$ & $\begin{array}{l}\text { No hay mecanismos previstos para hacer frente a } \\
\text { los riesgos laborales }\end{array}$ \\
\hline $\begin{array}{l}\text { Competencias laborales } \\
\text { específicas requeridas para } \\
\text { el puesto }\end{array}$ & $\begin{array}{l}\text { Se requiere tomar un curso básico de } 90 \text { horas } \\
\text { semanales, por tipo de población con la que se } \\
\text { pretende trabajar. }\end{array}$ & $\begin{array}{l}\text { Se ofrecen cursos dirigidos a personas de } 18 \text { años o } \\
\text { más, con primaria completa. }\end{array}$ \\
\hline $\begin{array}{l}\text { Valoración económica del } \\
\text { trabajo }\end{array}$ & $\begin{array}{l}\text { La remuneración máxima que puede brindar el estado } \\
\text { por } 80 \text { horas mensuales de trabajo asciende a } 15.000 \\
\text { pesos uruguayos, aproximadamente. Sin embargo, } \\
\text { este monto total es otorgado a aquellos hogares en } \\
\text { los que las personas ganen } 10.000 \text { pesos o menos per } \\
\text { cápita, mientras que en aquellos hogares donde las } \\
\text { personas ganan } 36.000 \text { pesos per cápita, el SNIC no } \\
\text { subvenciona el servicio. }\end{array}$ & $\begin{array}{l}\text { No se prevén mecanismos de valoración económica } \\
\text { del trabajo }\end{array}$ \\
\hline $\begin{array}{l}\text { Modificación de la división } \\
\text { sexual del trabajo en el } \\
\text { trabajo de cuidados }\end{array}$ & $\begin{array}{l}\text { El Plan de Formación se dirige a “las cuidadoras” para } \\
\text { reconocer la feminización del trabajo de cuidados, } \\
\text { aspecto que se ve como positivo desde el SNIC. }\end{array}$ & $\begin{array}{l}\text { No se explicitan mecanismos de fomento a la } \\
\text { participación de los varones (cuotas de cuidadores, } \\
\text { lenguaje que incluya a los varones). La reproducción } \\
\text { de las características que el sector de cuidados } \\
\text { ya posee, de informalidad, precarización y } \\
\text { poca remuneración del empleo, no mejora las } \\
\text { condiciones de vida de las mujeres cuidadoras } \\
\text { remuneradas ni genera un incentivo para la } \\
\text { participación de otras mujeres o varones en la tarea. }\end{array}$ \\
\hline
\end{tabular}

Fuente: Elaboración propia en base a los datos del Programa de Asistentes Personales de la página del Sistema Nacional de Cuidados. Referencias: http://www.mides.gub.uy/innovaportal/v/63068/3/innova.front/es-hoy y http://www.sistemadecuidados.gub.uy/innovaportal/v/61039/1/ innova.front/personas-en-situacion-de-dependencia 


\section{Reflexiones finales}

Dadas las características y malas condiciones laborales ligadas al trabajo remunerado de cuidados en domicilio en el marco del Programa $\mathrm{AP}$, no se visualizan incentivos para la retención y profesionalización de la fuerza de trabajo, aspecto que va en detrimento de la posibilidad de creación de un sector ocupacional de cuidados capaz de atraer no solo a las mujeres que buscan una fuente de trabajo de fácil acceso a raíz de que han sido expulsadas de otros sectores o no cuentan con experiencia o formación amplia o especializada previa, sino también a los varones.

La combinación entre las exigencias técnicas del trabajo - sobre todo aquellas ligadas a la invisibilidad de la dimensión emocional del trabajo-, la ausencia de mecanismos para prevenir los riesgos laborales, la baja formación requerida para el acceso al puesto de trabajo, junto con condiciones de trabajo malas (baja remuneración, ausencia de puestos intermedios u opciones laborales que permitan el ascenso profesional, entre las más destacadas), puede constreñir fuertemente la voluntad de superación a nivel profesional y promover el abandono del puesto de trabajo, incluso de aquellas trabajadoras fuertemente comprometidas con su trabajo y profesión.

En la medida en que la invisibilización de los requerimientos técnicos asociados al puesto de trabajo siga existiendo y los mecanismos de producción y reproducción de la desvaloración del trabajo femenino continúen operando, la gestión de los riesgos laborales y de la capacitación en el puesto de trabajo seguirá siendo asumidos de forma individual por quienes se ocupan de este tipo de empleo.

Esto, sumado a la ausencia de soporte técnico del Programa AP, son aspectos que van en detrimento de la calidad del servicio provisto a las personas que son sus usuarias, así como de la generación de condiciones de trabajo dignas del empleo de cuidados a domicilio. A su vez, hablan de la reproducción del paradigma médico donde el énfasis está puesto en la rehabilitación de las personas dependientes y no en una relación entre cuidado y cuidador horizontal, y contenido de manera técnica y comunitaria para garantizar los derechos de todas las partes al cuidado.

Visibilizar los requerimientos técnicos derivados del trabajo es un primer hito, necesario para identificar y reconocer las dimensiones y características del trabajo remunerado de cuidados a domicilio, los tipos de riesgos laborales asociados, el tipo de soporte técnico requerido a nivel organizacional y las necesidades de formación y capacitación de quienes acceden y se desempeñan en él.

Al respecto, emerge la pregunta sobre si el SNIC se está constituyendo como un sistema que genera servicios (es decir, que piensa en el soporte técnico, la formación, el vínculo de cuidados $-y$ sus dimensiones emocionales-, la infraestructura necesaria) o si indirectamente se orienta a las políticas de transferencias monetarias (directas o indirectas) para el cuidado en domicilio. Si bien el Programa de Asistentes Personales parecería tener componentes de servicio de cuidado (dirigido al ámbito doméstico) algunos de los resultados del análisis muestran que en varias dimensiones relevantes los planteamientos para ser concebido como un servicio de atención a la dependencia son escasos o nulos.

Como organismo rector de las políticas de cuidado, el SNIC tiene la posibilidad de instalar lógicas de trabajo que colaboren a la valoración y resignificación del trabajo de cuidados, así como contribuir a fomentar la participación de los varones. Si bien en el diseño programático se explicita que estos son objetivos del sistema, su realización está comprometida en el tipo de prestaciones (tanto monetarias como servicios) que efectivamente sean provistas o logren ser desarrolladas. Las características de las prestaciones diseñadas tienen un impacto diferencial tanto a nivel de la protección del derecho a ser cuidado como de las condiciones en las cuales el trabajo remunerado de cuidado a domicilio es llevado a cabo, lo cual a su vez tiene efectos para la reproducción o freno de los estereotipos y desigualdades de género. Se ha planteado en la literatura sobre los Estados de Bienestar y la organización social del cuidado que es importante mercantilizar algunos servicios con el fin de redistribuir la carga del trabajo de cuidados entre las familias y otros actores como el Estado, el mercado y la comunidad (Esping-Andersen, 2000). Sin embargo, a los efectos de que la corresponsabilidad incluya una perspectiva de derechos en el cuidado y una perspectiva de género, es fundamental que los procesos de tercerización velen por al menos dos cuestiones de base: que el trabajo de cuidados sea un trabajo que tenga valor social y que el trabajo de cuidados remunerado no reproduzca la división sexual del trabajo que coloca a las mujeres en lugares desventajosos en cuanto a la autonomía y el ejercicio de la ciudadanía social, con relación a los varones. Para esto, es necesario que las condiciones de trabajo sean dignas y permitan la profesionalización de la tarea, lo cual hace preciso que los elevados niveles de cualificación asociados a la tarea sean reconocidos y que las condiciones laborales sean acordes a ellos. Podría agregarse a estos dos un principio de universalización de los servicios, dado que los cuidados implican tensiones, aunque distintas, sobre las mujeres de todas las clases sociales (y no sobre sus pares varones).

Además, en el Programa AP se establece que quienes cuiden remuneradamente son asistentes “profesionales" pero ni las exigencias (formación específica en cuidados requerida para acceder al puesto de trabajo) ni las condiciones laborales del trabajo asegurarían un trabajo de calidad. Por tanto, no es claro el sentido de la palabra "profesional" a 
la que hace alusión el programa, dado el desajuste entre los elevados requerimientos técnicos del puesto de trabajo y las malas condiciones de trabajo propuestas por el Estado.

El punto de partida en el trabajo de cuidados es que carece de valor social, asociado al servilismo y a la naturalización de la actividad como condición de naturalmente femenina y realizada en el hogar. Uno de los indicadores de la desvalorización de este trabajo es que en Uruguay se realiza mayoritariamente de manera gratuita, es decir, que mediante él no se accede a un salario.
Así, la estrategia de ofrecer dinero para contar con un asistente personal por el Estado no estaría contribuyendo a que el cuidado se transforme en un trabajo valorado socialmente. En sintonía con lo que plantea Torns (2008), en el caso del SNIC y en particular del Programa AP, dadas las características y las malas condiciones de las asistentes personales, el Estado se encuentra más cerca de estar reproduciendo un "familismo implícito", reforzando los producción de roles de género presentes en la clásica división sexual del trabajo. 
ABBOTT, A. (1988): The system of proffesions, Chicago, The University of Chicago Press.

ADELANTADO, J. (1998): "Las relaciones entre estructura y políticas sociales: una propuesta teórica", Revista Mexicana de Sociología, no 3 , pp. 123-156.

AGUIRRE, R. (2003): Género, ciudadanía social y trabajo, Montevideo, Doble Clic Editoras.

- et al. (2009): Las bases invisibles del bienestar social. El trabajo no remunerado en Uruguay. Montevideo, Doble Clic Editoras.

- (2013): Personas ocupadas en el sector cuidados. Sistema Nacional de Cuidados. Montevideo.

- et al. (2014): "Los cuidados en la agenda de investigación y en las políticas públicas en Uruguay", Íconos. Revista de Ciencias Sociales, no 50, p.43-60.

BATTHYÁNY, K. (2015): “Los tiempos del cuidado en Uruguay” en Batthyány, K. (Ed.) Los tiempos del bienestar social. Género, trabajo no remunerado y cuidados en Uruguay, Doble Clic Editoras, pp.87-132.

BATTHYÁNY, K; GENTA, N. y PERROTTA, V. (2013): La población uruguaya y el cuidado. Análisis de representaciones sociales y propuestas para un sistema de cuidados en Uruguay, Serie Sistema Nacional de Cuidados.

BATTHYÁNY, K; GENTA, N. y PERROTTA, V. (2017): “El aporte de las familias y las mujeres al cuidado no remunerado en salud en Uruguay", Revista Estudios Feministas, noㅡ 25 (1), pp. 187-213.

BATTHYÁNY, K; GENTA, N. y SCAVINO, S. (2016): Análisis de género de las estrategias de cuidado infantil en Uruguay. En Boado, M (Coord.): El Uruguay desde la sociología 14, Doble Clic Editoras.
BAZO, M.T. (1998): "Vejez dependiente, políticas y calidad de vida", Revista Papers, nํ5, pp. 143-161.

BOCK, G. y DUDEN, B. (1985): "Trabajo por amor, amor como trabajo", Desarrollo, Revista de la Sociedad Internacional para el Desarrollo, vol. 2, p. 4-14.

BANCO DE PREVISIÓN SOCIAL (2015): Encuesta longitudinal de protección social, Ronda 2012-2013, Montevideo, BPS.

BROTHERIGE, C. y GRANDEY, A. (2002): “Emotional Labour and Burnout: Comparing Two Perspectives of 'People Work'”. Journal of Vocational Behavior, no 60, pp.17-39.

CANCIAN, F. y OLIKER, S. (2002): Caring and Gender, Oxford, Altamira Press.

CALVO, J.J. et al. (2013): “Las necesidades básicas insatisfechas a partir de los Censos 2011", Atlas Sociodemográfico y de la desigualdad en Uruguay, Trilce.

CAMERON, C. y MOSS, P. (2007): Current understandings and future directions, Routledge.

CARRASCO, C.; BORDERÍAS, C. y TORNS, T. (2011): El trabajo de cuidados. Historia, teorías y política, Los libros de la catarata.

CEPAL (2014): “La medición del tiempo y el aporte de las mujeres a la economía”, Notas para la Igualdad, $\mathrm{n}$ - 15 , Observatorio de igualdad de Género de América Latina y el Caribe.

CHODOROW, N. (1978): The reproduction of Mothering, University of California Press.

DALY, M. y LEWIS, J. (2000): "The concept of social care and the analysis of contemporary welfare states", en British Journal of Sociology, $\mathrm{n}-51$, pp. 281-298. 
DALY, M. y LEWIS, J. (2011): “El concepto de 'Social Care' y el análisis de los Estados de Bienestar contemporáneos”, en Carrasco, C. Borderías, C. y Torns, $\mathrm{T}$ (eds.) El trabajo de cuidados. Historia, teorías y política, Madrid, Los libros de la catarata.

DAVIES, C. (1995): "Competence versus Care? Gender and Caring Work Revisited”, Acta Sociológica, 38.

DURÁN, M.A. (2006): “Dependientes y cuidadores: el desafío de los próximos años”, Revista del Ministerio de Trabajo e Inmigración, n. 6o, p. 57-66.

ENGLAND, P. (2005): “The emerging theories of care work", Annual Review of Sociology, , n. 31, p. 381-399.

ESCUDERO, B. (2007): "Las políticas sociales de dependencia en España”, Revista Española de Investigaciones Sociológicas, 119/07, p. 65-89.

ESPING ANDERSEN, G. (2000): Fundamentos sociales de la economías postindustriales, Ariel Sociología.

GÓMEZ BUENO, C. (1999): “Del etiquetado de las ocupaciones según nivel de cualificación”, Revista de Investigaciones Sociológicas, $\mathrm{n} \div 08$, p. $33-62$.

GRAHAM, H. (1983): Caring, a labour of love: woman, work and caring, Routledge- Kegan Paul.

HOCHSCHILD, A. (1983): The managed heart. Commercialization of human feeling. University of California Press.

HOCHSCHILD, A. (1995): "The culture of politics: traditional, posmodern, cold-modern and warm-modern ideals of care”, Social Politics, no 3, p. 331-346.

JIMÉNEZ, A. y HUETE, A. (2010): "Políticas públicas sobre discapacidad en España. Hacia una perspectiva basada en los derechos", Política y Sociedad, 47, n- 1 , p. 137-152.

INAU (2009). Estrategia nacional para la infancia y adolescencia. Bases para su implementación, INAU.

INE (2013): Uso del tiempo y trabajo no remunerado en Uruguay. Principales resultados de la encuesta de Uso del Tiempo 2013, INE.

INMUJERES (2013): Estadísticas de género 2013. Evolución de los indicadores de género en el período 2009-2013, Ministerio de Desarrollo Social.

KAPLAN, A. (1987): “Invisible Work”, Social Problems, n- 34, pp. 403-415.

MARTÍNEZ BUJÁN, R. (2010): “La reorganización de los cuidados familiares en un contexto de migración internacional", Cuadernos de Relaciones Laborales, 29, nㅡㄴ 1, p. 93-123.

MARTINEZ, J. y VOOREND, K. (2013): “Desigualdades de género en los regímenes de bienestar latinoamericanos: mercado, política social y organización familiar de los cuidados", en PAUTASSI, L. y ZIEBECCHI, C. (eds.), Las fronteras del cuidado. Agenda, derechos e infraestructura, Editorial Biblos.
MIDES (2014): Cuidados como sistema. Propuesta para un modelo solidario y corresponsable de cuidados en Uruguay, Ministerio de Desarrollo Social.

ORTEGA, C. y LÓPEZ, F. (2004): “El burnout o síndrome de estar quemado en los profesionales sanitarios. Revisión y perspectivas", International Journal of Clinical and Health Psychology, $\mathrm{n}-1$, vol. 4.

PAREDES, M. et al. (2010): Indicadores sociodemográficos de envejecimiento y vejez en Uruguay: una perspectiva comparada en el contexto latinoamericano, Lucida Ediciones.

PICKARD, L. (2015): “A growing care gap? The supply of unpaid care for older people by their adult children in England to 2032", Ageing and Society, $\mathrm{n}-35$ (1), p. 96-123.

RECIO, C. (2014): El empleo en el sector de atención a las personas en España, tesis doctoral, Departamento de Sociología, Universitat Autònoma de Barcelona.

RISMAN, B. (2004): "Gender as a social structure. Theory Wrestling with Activism", Gender \& Society, 18, no 4 , p. 429-450.

RODRIGUEZ CABRERO, G. (2011): "Políticas sociales de atención a la dependencia en los Regímenes de Bienestar de la Unión Europea", Cuadernos de Relaciones Laborales, 29, n- 1, p. 13-42.

SIVE (2015): Las personas mayores en Uruguay: un desafío impostergable para la producción de conocimiento y las políticas públicas, INMAYORES-DINEM.

SNIC (2015): Plan Nacional de Cuidados 2016-2020, MIDES Junta Nacional de Cuidados, 2015

TORNS, T. (2015): “El trabajo y el cuidado: cuestiones teórico-metodológicas desde la perspectiva de género", Empiria. Revista de Metodología de Ciencias Sociales, № 15, pp. 53-73.

- et al. (2009): "Temps i cura: la coresponsabilitat social de la cura a la vida quotidiana” en BRULLET, C. (ed.), Temps i cura: la coresponsabilitat social de la cura a la vida quotidiana, Generalitat de Catalunya, Departament d'Acció Social i Ciutadania, Secretaria de Polítiques Familiars i Drets de Ciutadania.

- et al. (2014): Nuevas profesiones para la organización social del cuidado cotidiano. Estudios e investigaciones, Universidad Autónoma de Barcelona.

VAN ROMPAEY, E. (2015): Cualificación invisibilizada. Estudio sobre el trabajo remunerado de cuidados en domicilio en los servicios de atención barcelonés y montevideano. Tesis Doctoral, Departamento de Sociología, Universidad Autónoma de Barcelona.

WAERNESS, K. (1984): “The Rationality of Careing”, Economic and Industrial Democracy, $\mathrm{n}-5$, p. 187-211.

ZAPF, D. (2002): "Emotion Work and Psychological Wellbeing. A Review of the Literature and Some Conceptual Considerations", Human Resource Management Review, no 12, p. 237-268. 